\title{
A summary of the CIMOSA reference architecture
}

\author{
J. Vlietstra
}

\subsection{CIMOSA AND ITS ASSOCIATED CONSTRUCTS [1]}

The ESPRIT project AMICE $^{1}$ has developed an Open System Architecture (CIMOSA) for the manufacturing industry. The main goal of CIMOSA is to support process oriented modelling of manufacturing enterprises and to provide execution support for operation of enterprise systems based on those models. Emphasis in CIMOSA is on modelling support for business users ${ }^{2}$ rather than for modelling specialists.

\subsection{ENTERPRISE INTEGRATION - THE SOLUTION}

Enterprise integration has to provide for flexibility of enterprise operations and efficient use of enterprise assets. Therefore, a modular approach to integration is the most promising one. This is an approach which models the operation as a set of cooperating processes exchanging results and requests (events) between themselves. Only the exchanged objects need to have a representation which is common between partners. This minimal unification of objects, together with an integrating infrastructure for hiding heterogeneity, can readily cope with enterprise integration. The CIMOSA approach enables integration of both reengineered and existing systems.

1. AMICE: European Computer Integrated Manufacturing Architecture (reverse acronym)

2. Business users (or business professionals) are all those people with responsibilities for particular business processes. Business users are: technical staff, administrative staff and all levels of management. 


\subsection{MANAGEMENT OF CHANGE}

Companies are not always organized for the use of fast decision making processes. Departments are still managed according to their own subgoals rather than working toward the overall enterprise objectives. Responsibilities are still structured in one dimensional hierarchies which mix responsibilities for enterprise assets with those for enterprise operations. Matrix organizations are still a rather theoretical concept. In addition, the decision making process in many companies is still based on traditional information processing, that is, information gathering with 'paper and pencil,' on request and from inconsistent sources. This process is defined from the view of a rigid management hierarchy and is very prone to severe information losses. The process is very time consuming and often yields only insufficient or even wrong information and decisions. To achieve 'real time' operational flexibility, delegation of responsibility and authority are needed to allow people to make changes as they are required rather than going through a hierarchy of many levels for decision sign-offs. Ease of access and more efficient use of information will enable the delegation of more responsibility and authority.

Therefore, active management of change is the most significant future requirement for successful enterprise operation. This means recognizing and reacting to external changes as early as possible and defining and implementing the internal modifications to their response. Business process reengineering and simplification as tasks of enterprise engineering are prerequisites for enterprise integration.

Decision support is an essential part of the management of change. This implies a timely access to the right information and the distribution of the decision making process results to the right places. Decision making requires clear and explicit knowledge about the relevant business processes and their contents (information, resources, responsibilities and authorities), a definition of alternative solutions, and an analysis of their impact on the total enterprise operation. Therefore computer simulation of potential solutions is a requirement for future decision support systems.

CIMOSA provides decision support through its up-to-date models (identification of relevant information, analysis of alternative solutions and propagation of verified decisions) and ease of business process reengineering. 


\subsection{SCOPE OF ENTERPRISE INTEGRATION}

Industry needs an implementation strategy in which an existing, semiautomated enterprise can steadily progress in discrete steps towards CIM. The manufacturing plant must continue to operate day by day, but, at the same time, incorporate changes in the direction of both automation and integration. Since it is impossible to purchase all of the components required for realizing an enterprise environment from a single vendor, standards are required to insure the correct interworking of components from multiple sources.

In addition to its capital investments, the enterprise's knowledge about its business and technical processes is one of its most valuable assets, often differentiating it from its competitors. The integration of this internal knowledge with the external one arising from other sources (e.g. trade associations, de facto standards, formal standards) is vital for an enterprise trying to remain competitive and cost effective.

Enterprise operation also consists of internal and external parts. Internal operation of an enterprise is that set of business processes needed to market, develop, manufacture, distribute and sell products and to administer and manage the operation itself. External operations which are coupled to the internal ones cover relationships with suppliers, customers, financial institutions, government agencies, etc. The main focus of enterprise integration is on internal environments. However, relations to the external environments and their impact on the internal operation have to be made visible as well. Only if these dependencies are known and have become part of the business model can the impact of changes be fully evaluated. However, more detailed modelling is required for internal operations than for external ones. Whereas the control flow of internal business processes is needed even for simulation of alternatives, identification of shared information and dependencies is mostly sufficient for modelling the external relations.

Information technology hardware makes it possible even today to install very large networks of computer systems with an almost unrestricted performance and processing capability. However, the ability to handle the vast amount of information needed and existing in the manufacturing enterprise and to process the right information, for the right purpose, at the right time and in the right place is still a major problem.

CIMOSA provides means to describe in a consistent way both internal and external enterprise operations and their information needs. In addition, it clearly distinguishes between model engineering and model use and defines in its system life-cycle a formal engineering release for operation. 


\subsection{ENTERPRISE ENGINEERING AND OPERATION}

Enterprise evolution leads to a continuous need for enterprise integration. This is a need to be fulfilled by new engineering tasks concerned with maintaining and extending enterprise operation efficiency and flexibility. Therefore, enterprise integration has to be conceived as a modular approach structuring and modelling the business into manageable units (processes) which cooperate with each other according to identified needs and sharing information on request. Only with the enterprise modelled as a set of cooperating processes rather than as a large monolithic entity can changes be implemented and operational modifications and extensions be made in 'real time.' Only then can sufficient operational and organizational flexibility be achieved.

Information Technology (IT) based integration support for business model engineering provides:

1. Definition of business process flow of control and information needs.

2. Definition and organization of enterprise assets (resources and information).

3. Definition and organization of enterprise responsibilities and authorities.

4. Maintenance of business models (modifications and extensions) by the business user.

The set of business processes which make up the enterprise operation have to operate and interoperate in a highly efficient and flexible manner. To assure operational flexibility and efficiency an easily accessible knowledge of the operation has to be provided through up-to-date business models. Better structuring and modelling of the enterprise operation will hide process complexity and thereby improve decision making and business management.

Operational flexibility will be greatly enhanced by the direct use of models for operational control and monitoring. Business changes will be implemented and validated in the model and directly released for operation. Improved enterprise logistical control and superior asset management will also contribute to operational flexibility and efficiency.

IT based integration support for business model execution provides:

1. Evaluation of impact of change and alternative solutions (model simulation).

2. Model based operation control and monitoring. 


\subsection{COEXISTENCE WITH HERITAGE/LEGACY SYSTEMS}

New system components, reengineered according to new paradigms in modelling still have to interoperate with the existing parts of the enterprise operation. With an average life time of about 12 years for Mainframe applications [2] there will be many years of such coexistence.

Therefore, any new methodology and technology used in enterprise operation has to provide means for interoperating with the existing world. The most promising approach to achieve interoperability between incompatible systems is through the use of information objects shared between different parts of the system. With this approach agreement on common representation is only necessary between partners sharing the same objects. This enables the identification of classes of information objects according to their degree of sharing in terms of private, common or public use. This is the approach taken also by people working in artificial intelligence (AI) and on the development of ontologies (generic theories of common aspects of reality).

CIMOSA provides solutions for the enterprise integration of both reengineered and heritage systems.

\subsection{BUSINESS BENEFITS}

Enterprise reengineering and process simplification are tasks which will coordinate enterprise evolution as well as improving enterprise performance in general. In the IT arena the availability and sharing of information between different business areas as well as the common use of computer services will reduce operations costs, but more importantly it will improve operational quality and flexibility. Providing relevant information in real time to the decision makers will enable a faster reaction to different market changes (markets on goods, services, knowledge, technology and money). It will also help to base enterprise strategies on real life facts rather than fictions.

CIMOSA will provide benefits for enterprises by:

1. Improving enterprise operational flexibility and efficiency by the reengineering and simplification of business processes.

2. Supporting the management of change by evaluation of alternatives through the simulation of operations.

3. Improving operational flexibility and efficiency and reducing operational costs through better business management (people, process, resources, information). 
4. Reducing lead times through the sharing and reuse of relevant information, modelling building blocks and system components.

\subsection{ENTERPRISE MODELLING AND ITS REQUIREMENTS}

Enterprise business modelling is a prerequisite for successful enterprise integration [3]. However, enterprise operations have to be well understood and explicitly described and presented in order to identify inconsistencies and incompatibilities and to analyze their consequences. Alternative solutions can then be modelled and evaluated through simulation. Also enterprise modeling has to meet the requirements of a number of different users in their day-to-day operations. Therefore modelling has to be based on business objectives and must describe the operation in terms of its related functionality and dynamic behavior (control flow).

Enterprise modelling should not be done as a one time all encompassing venture. A modular structure will allow for evolutionary model building and model maintenance. However, to assure consistency, all modules have to be parts of, derived from and linked to a common model. These submodels must meet specific user needs for optimising and structuring certain aspects of the operation without being constrained by a huge model. Levels of abstraction are needed to support strategic, tactical and operational planning and decision making. Again, all levels have to be abstractions from the same underlying model.

Model engineering needs heavy IT support to enable the creation of consistent and easily maintainable and extendible models. The users have to be guided through the model engineering process by providing them with an identification of those reusable building blocks already known to the system. Ease of maintenance is needed to adapt models in real time to the changing internal and external environment as well as to enable the evaluation of alternatives to the existing situation ('as-is' versus 'to-be' models).

Unlike other approaches CIMOSA allows the users to model different parts of the enterprise separately and to integrate them later. CIMOSA provides a modular approach (domain process) for business modelling by identifying three modelling levels (requirements definition, design specification and implementation description) and four views (function, information, resource, organization) as part of an open set. 


\subsection{AMICE PROJECT}

The goal of the AMICE project is to develop an Open System Architecture for CIM (CIMOSA) which will facilitate continuous enterprise evolution and make needed operational improvements manageable while at the same time providing a strategy to protect current and planned investment in an enterprise's asset base. Such an architecture will become the base for consistent and maintainable enterprise engineering and operation support and thereby be the cornerstone for an industrial infrastructure for industry wide use of information and supporting technology.

CIMOSA provides a consistent enterprise model building and maintenance methodology and an integrating infrastructure for model engineering and execution support. CIMOSA is based on a process oriented modelling approach describing all enterprise activities in a common way. Such activities include manufacturing processes on the shop floor as well as management and administrative processes.

Validation of the concepts has been carried out in various case studies in consortium member companies. The work has led to inputs into the standardization of the modelling and integrating infrastructure. The latter takes into account current work on standards like OSI (Open System Interconnection), ODP (Open Distributed Processing), etc. Standardization has been achieved for the Modelling Framework by a European PreNorm [4] which has become the base for the work on international standardization in ISO TC 184/SC5/WG1. Project results are summarized in 'Open System Architecture for CIM' [5], several publications by project members [6] and a recent private publication by the ESPRIT Consortium AMICE [7].

CIMOSA is a prenormative development effort aimed at guiding and supporting users and vendors in enterprise integration as well as in the development of compliant products which will ease enterprise integration. It will be usable in an evolutionary mode starting with enterprise model engineering and decision support and moving gradually into new modes of enterprise operation and management. To further verify CIMOSA goals and objectives the project concentrates on feasibility studies of the implementations of CIMOSA models and the Integrating Infrastructure which prepare the project for a future implementation of an industrial pilot.

\subsection{CIMOSA APPROACH}

CIMOSA provides a framework for guiding CIM users in enterprise system design and implementation, and CIM vendors in system component 
development. It provides a descriptive, rather than a prescriptive methodology supporting the system life-cycle. CIMOSA does not attempt to provide a standard architecture to be used by the whole manufacturing industry, but rather a reference architecture from which particular architectures can be derived which will fulfill the needs of particular enterprises.

The reference architecture provides constructs for the structured description of business requirements and for CIM system design and implementations. CIMOSA compliant enterprise systems will support organizational and operational flexibility, extensive use of multidisciplinary enterprise information (knowledge) and graceful system integration.

Through the business modelling framework a generic modelling concept is provided which is applicable to enterprises in many industries. This is a modelling concept which will vastly enhance communication and interoperability via Information Technology within an enterprise, as well as between different enterprises. Model execution in heterogeneous manufacturing and IT environments is supported by the implementation of an integrating infrastructure.

CIMOSA supports new paradigms in enterprise management, thus enabling the explicit description of enterprise processes at different levels of abstraction for use for strategic, tactical and operational decision support. Decision support is developed through the extensive simulation of alternatives and through the evaluation of decision impacts on all relevant parts of the enterprise. Specific definition of responsibilities and authority will allow for the deferring of decisions in time and for the reduction of the number of management levels in order to enable a flexible and efficient enterprise operation.

Applying CIMOSA modelling methodology results in complete descriptions of enterprise domains and of their contained business processes including their relationships to external agencies (suppliers, customers, government regulatory bodies, etc). This enterprise model is stored on and manipulated by the relevant information technology base of the enterprise. CIMOSA allows the modelling of the enterprise to be done incrementally rather than by following an overall top-down approach. It structures the enterprise operations into a set of interoperating domain processes exchanging results and requests. Several different views of the manufacturing enterprise content and structure are required to satisfy the needs of the different users of such an architecture. CIMOSA provides the necessary constructs to enable these multiple views to be created and manipulated by those users who have specialist knowledge of their particular field but who are not experts in IT. 


\subsection{SYSTEM LIFE-CYCLE}

Enterprises are very complex undertakings which can be rendered more manageable by structuring their complex operational domains into a set of interoperating modules and its time domain according to a life-cycle concept. CIMOSA employs the concept of domain and business processes for its modular structuring and those of system and product life-cycle for the time domain.

Product life-cycle is a well known notion which is used to structure the different phases of a product's life. Within a given manufacturing enterprise many such product life-cycles exist - one for each product - which gives rise to a set of overlapping life-cycles as shown in Fig. 6.1. In order that the tasks associated with each step of the product life-cycle may be executed in a controllable manner, enterprises define the procedures to be followed. These normally take the form of company standards, work instructions, process sheets, etc. Different parts of the organization may be responsible for different phases. For example 'use' and 'maintain' is normally a 'client' rather than a 'supplier' task.

This principle of the life-cycle can be extended to the enterprise system or parts thereof (domains). While today's business processes are in operation, their successors may be in the process of implementation. Strategic

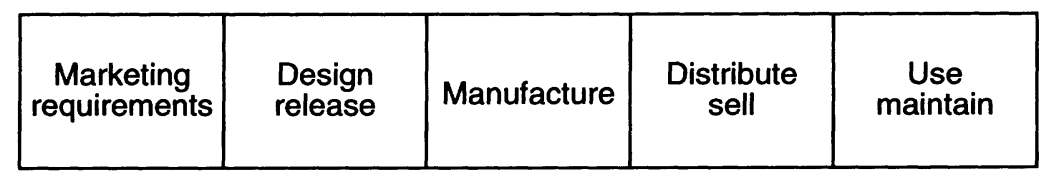

Product $\mathrm{A}$

\begin{tabular}{|c|c|c|c|c|}
\hline $\begin{array}{c}\text { Marketing } \\
\text { requirements }\end{array}$ & $\begin{array}{c}\text { Design } \\
\text { release }\end{array}$ & Manufacture & $\begin{array}{c}\text { Distribute } \\
\text { sell }\end{array}$ & $\begin{array}{c}\text { Use } \\
\text { maintain }\end{array}$ \\
\hline
\end{tabular}

Product B

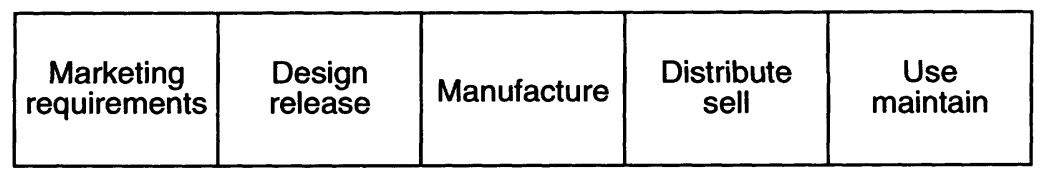

Product C

Figure 6.1 Overlapping product life-cycles. 


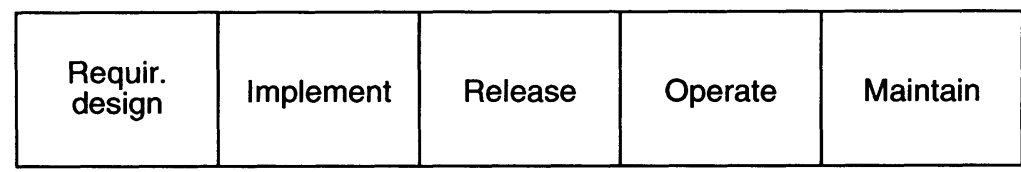

Enterprise

system

Figure 6.2 System life-cycle.

business plans under development today will set the stage for the systems implementations of tomorrow. Thus in an analogous way to products, the enterprise system which creates those products goes through a system lifecycle of development and use (operation) as shown in Fig. 6.2.

The tasks of the enterprise system life-cycle are concerned with the definition, description, creation and updating of the procedures and system components which govern and support the tasks of the product life-cycle. Thus product and system life-cycles are intimately interrelated. This relationship between the product and system life-cycles is summarized in Fig. 6.3. Whenever it is required to define a new or revised process supporting some part of a product life-cycle it will be necessary to carry out the tasks belonging to one or more phases of the associated system life-cycle. The operational phase of the system life-cycle is the execution of the relevant product life-cycle phases themselves. Thus by assigning enterprise tasks to one or the other of the life-cycles it is possible to structure the workings of the enterprise into enterprise engineering and operation and so render more effective the management of change.

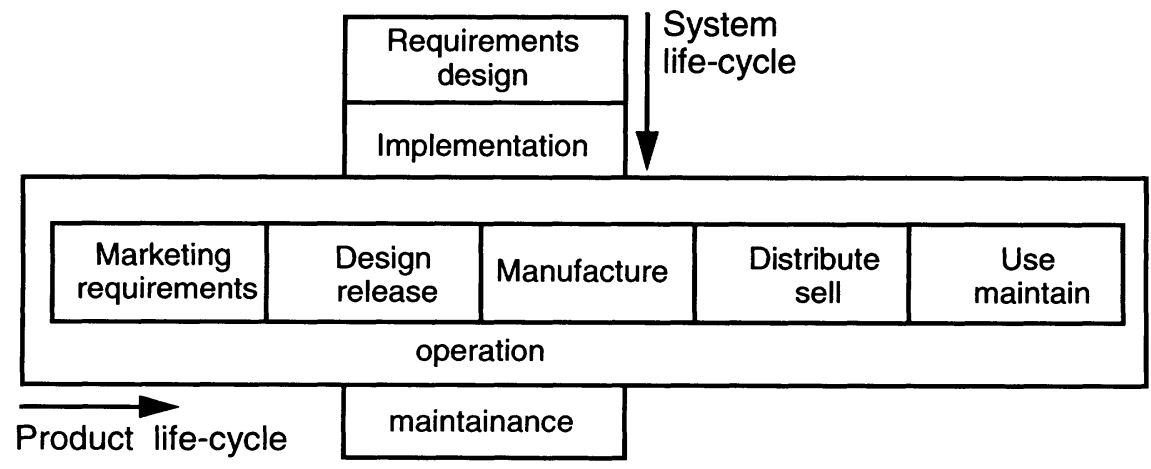

Figure 6.3 Relationship between system and product life-cycle. 


\subsection{ENTERPRISE INTEGRATION}

From the foregoing it will be recalled that enterprise integration has to be an ongoing process rather than a one time effort. The level of integration should remain a managerial decision and should be open to change over a period of time. Hence, one could find in some parts of a CIM enterprise a set of tightly coupled systems and elsewhere a set of loosely coupled systems according to the choices made by this particular enterprise.

The need to implement multivendor systems both in terms of hardware and software and of easy reconfigurability requires the provision of standard interfaces. To solve the many problems of the industry, integration has to recognize this and proceed on more than one operational aspect. The AMICE project identifies, and addresses, three levels of integration covering the aspects of physical systems, of application and of business integration (see Fig. 6.4).

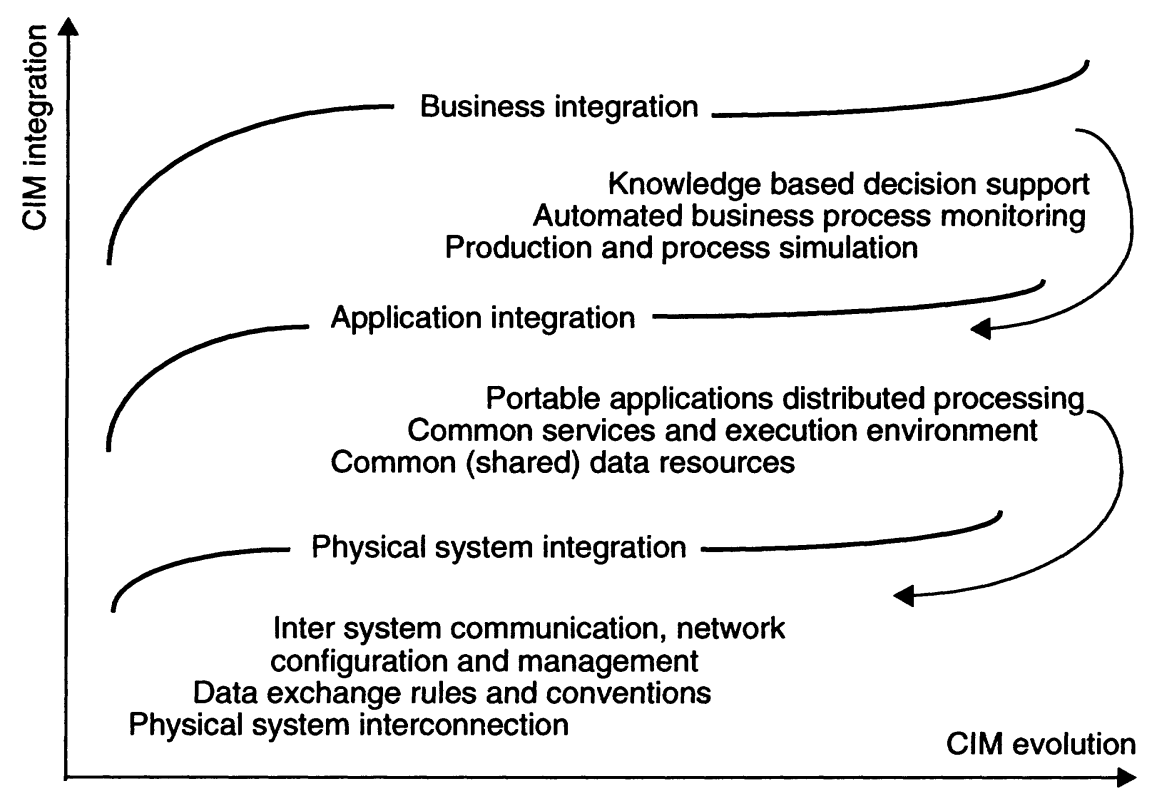

Figure 6.4 The levels of enterprise integration. 


\subsubsection{Business Integration}

Business Integration is concerned with the integration of those functions which manage, control and monitor business processes. These are functions which provide for supervisory control of the operational processes and in turn coordinate the day-to-day execution of activities at the Application level. The modelling of business processes and of their interrelations and the use of the resulting models for decision and operational support is the key to business integration.

\subsubsection{Application Integration}

Application Integration is concerned with the control and integration of applications (applications in the dataprocessing sense). This means there has to be interoperation between applications and users (human beings as well as machines) and the supply and removal of information through inter and intra system communication. Integration at this level means providing a sufficient Information Technology infrastructure to permit a system wide access to all relevant information regardless of where the data reside.

\subsubsection{Physical System Integration}

Physical System Integration is concerned with the interconnection of manufacturing automation and data processing facilities (that is, join CAD to CAM, provide for flexible manufacturing cells, implement computerized scheduling, etc.) to permit the interchange of information between the so called 'islands of automation' (inter system communications).

Dictated by the needs of the individual enterprise this interconnection of physical systems was the first integration requirement to be recognized and fulfilled. This need has led to a number of information technology concepts becoming well accepted standards in this area ${ }^{1}$.

1. For example: OSI (Open System Interconnection); MAP (Manufacturing Automation Protocol - an OSI implementation for the manufacturing shop floor). 


\subsection{CIMOSA OVERVIEW}

To satisfy the above issues of Management of Change, of Flexibility and of Enterprise Integration, CIMOSA provides three interrelated concepts:

1. Modelling Framework (Reference Architecture, Particular Architecture, Enterprise Model).

2. System life-cycle and Environments (Engineering and Operation).

3. Integrating Infrastructure.

CIMOSA recognizes previous efforts in enterprise integration especially in the manufacturing industry and draws from the experience already gained in enterprise modelling and computer systems integration. Some of the efforts which have been of particular importance for the development of enterprise integration are given in Reference [8].

\subsection{CIMOSA MODELLING FRAMEWORK}

The CIMOSA Modelling Framework provides the necessary guidance to enable end users to model the enterprise and its associated CIM system in a coherent way. The CIMOSA modelling approach is based on a reference architecture from which Particular Architectures and Enterprise Models can be developed. The structuring and decoupling of user concerns from the implementation constraints provided by the framework contributes to enterprise flexibility.

The Modelling Framework provide a structure which clarifies the relationships between the parts which make up the enterprise operational system (Information Technology and Manufacturing Technology Components) and the methods and software tools which are required to describe, simulate and operate such an industrial system.

When modelling an enterprise there are many aspects and viewpoints to be examined that cannot be structured in a one dimensional framework. CIMOSA identifies a three dimensional framework offering the ability to model different aspects and views of an enterprise (see Fig. 6.5 ):

1. The genericity dimension (Building Blocks) is concerned with the degree of particularisation. It goes from generic building blocks to their aggregation into a model of a specific enterprise domain. This dimension differentiates between the Reference and Particular Architectures.

2. The modelling dimension provides the modelling support for the system life-cycle starting from statements of requirements to a description of the system implementation. 

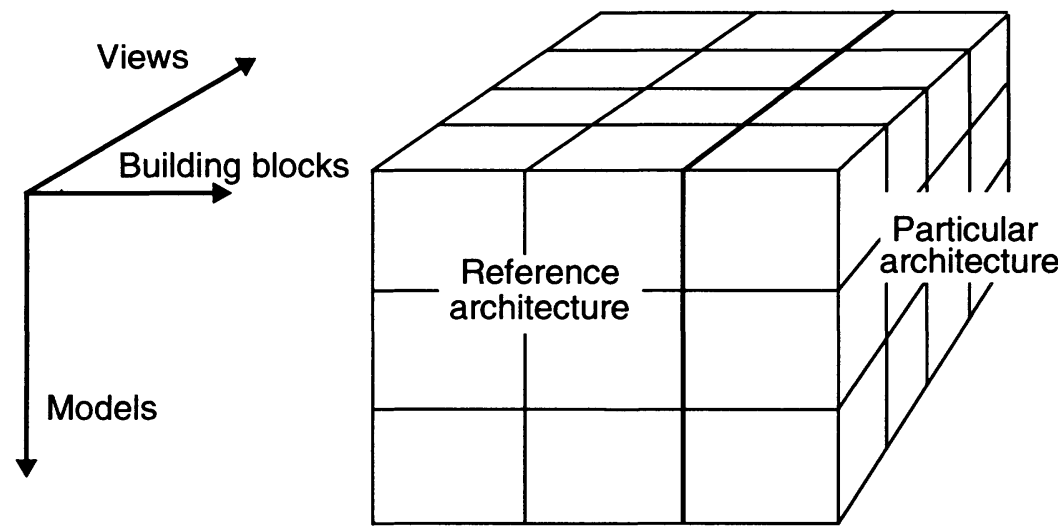

Figure 6.5 The CIMOSA modelling approach.

3. The view dimension is concerned with system behavior and functionality. This dimension allows the user to work with submodels representing different aspects of the enterprise (function, information, resource, organization).

\subsubsection{CIMOSA reference architecture}

Within the Modelling Framework, CIMOSA provides the CIMOSA Reference architecture. It resembles a catalogue of reusable building blocks which contains generic building blocks and aggregations of generic building blocks (Partial Models) applicable to specific modelling needs. See Fig. 6.6. User Oriented Generic Building Blocks are provided for:

Behaviour

Function

Information

Resources
Domain and Business Process, Event, Procedural Rules, Objective, Constraint

Enterprise Activity, Functional Operation (input/output for function, control, resource), Objective, Constraint

Enterprise Object, Object View, Information Element, Resources:

Capability, Functional Entity, Resource Unit, Cell 
Organization:

Responsibility, Authority, Organizational Unit, Organizational Cell

IT Oriented Generic Building Blocks complement user building blocks supporting IT system design and implementation description. Such building blocks are Schemas, Transactions, Configurations and specific Functional Entity types representing the Integrating Infrastructure.

Partial Models are aimed at particular types of enterprise domains or even types of enterprises. Such partial models are created using the set of generic building blocks. Such partial models will be provided by vendors to be used as macros to enhance the model building process and as implementable components for system implementation.

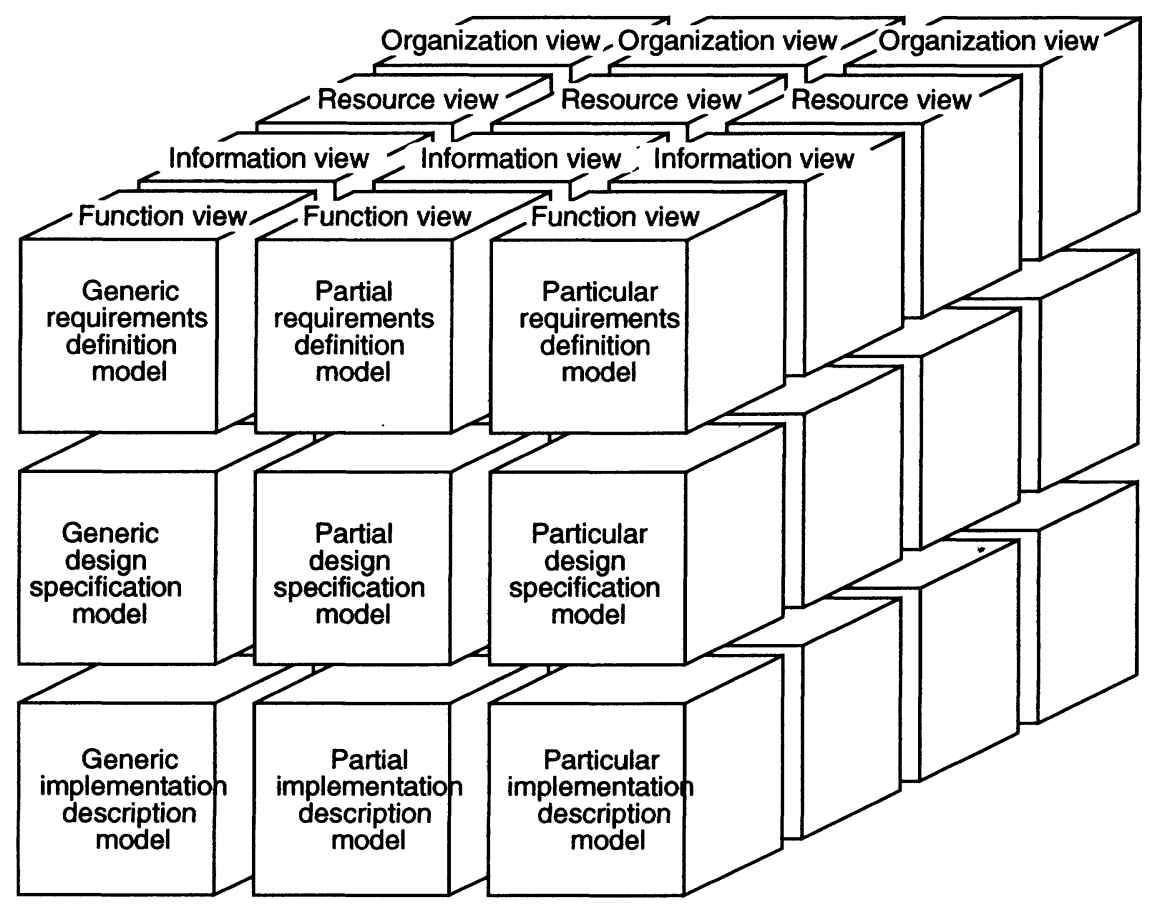

Figure 6.6 Overview of the CIMOSA architectural framework. 


\subsubsection{CIMOSA particular architecture}

The structuring of particular enterprise domains follows the framework of modeling levels and views. In the Process/Activity/Operation approach an enterprise is seen as a collection of interrelated but nonoverlapping domains, which are subsets of the enterprise business. Enterprise engineering is concerned with requirements, design and implementation of enterprise operations.

Enterprise operations are defined in two distinct levels linked to each other by events:

1. A network of event driven cooperating domain processes

2. An explicit description of the functionality and behavior of the individual domain processes presented as a network of enterprise activities/functional operations initiated by events and driven by an explicit set of procedural rules.

The network of domain processes may consist of CIMOSA and nonCIMOSA domain processes, that is, domain processes with or without an internal CIMOSA structure. This approach provides a potential solution for the enterprise integration of heritage systems. Figures 6.7 and 6.8 represent the two levels of such structuring. Fig. 6.7 shows a set of domain processes cooperating on shared objects (results and events). One of those processes is detailed in the following figure demonstrating the engineering process of decomposition (top-down) leading to a network of enterprise activities. It also shows the use of function aggregation (bottom-up) into Business and domain processes. Decomposition can be either into business processes representing lower level behavior or enterprise activities representing functionality. For a system design enterprise activities can be further decomposed into functional operations which relate this functionality to enterprise resources for execution.

\subsubsection{CIMOSA enterprise model}

According to the structure provided for the particular architecture, CIMOSA models capture business knowledge globally as networks of domain processes and also as enterprise activities representing detailed local functionality and as business processes representing intra process behavior. Identification of all the inputs required and the outputs produced (information, control, resource and organizational) will be done for each of the different parts of this functionality. Modelling is done through the instantiation of generic building blocks or partial models. 


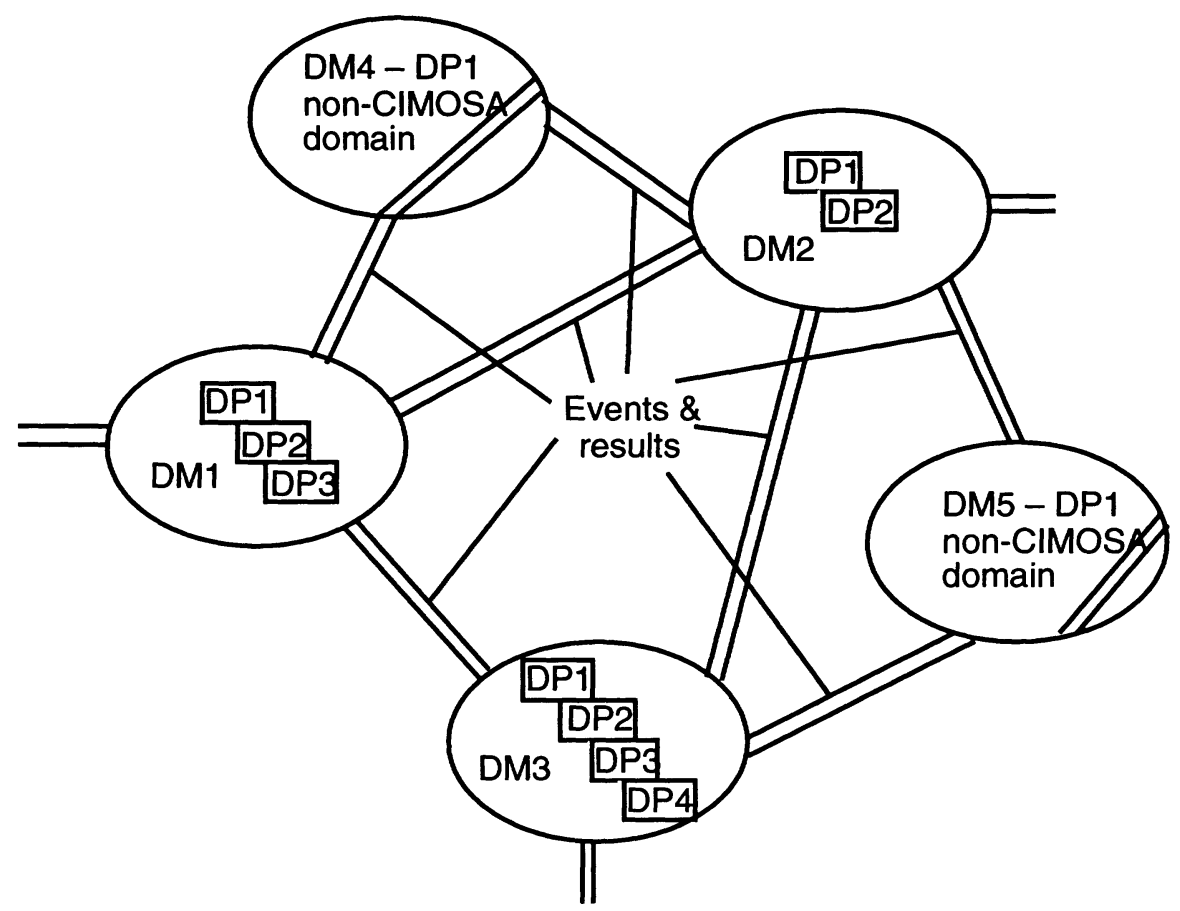

Figure 6.7 Network of domain processes ( $\mathrm{DM}=$ domain, $\mathrm{DP}=$ domain process).

Within an enterprise, tasks (domain processes) are usually organized into subtasks (business processes, enterprise activities, functional operations) which need to be realized to achieve the business objectives. Within each domain (unsolicited) real world happenings and requests (events) to do something will occur which will trigger domain processes. Domain processes can also have exchange with domains external to the enterprise. To represent tasks and actions performed within an enterprise CIMOSA forces users to think in terms of 'processes', 'activities' and 'operations', where operations define the lowest level of granularity to represent tasks performed within an enterprise. The level of detail to be described in the model is at the users discretion and not dictated by CIMOSA.

Model modifications can be made via a domain process' internal and external behavior and its functionality. Internal changes will be made through modification of internal control flow and/or functionality, changes in external behavior will be modelled through modifications of its external relations (interoperation with other domain processes). 


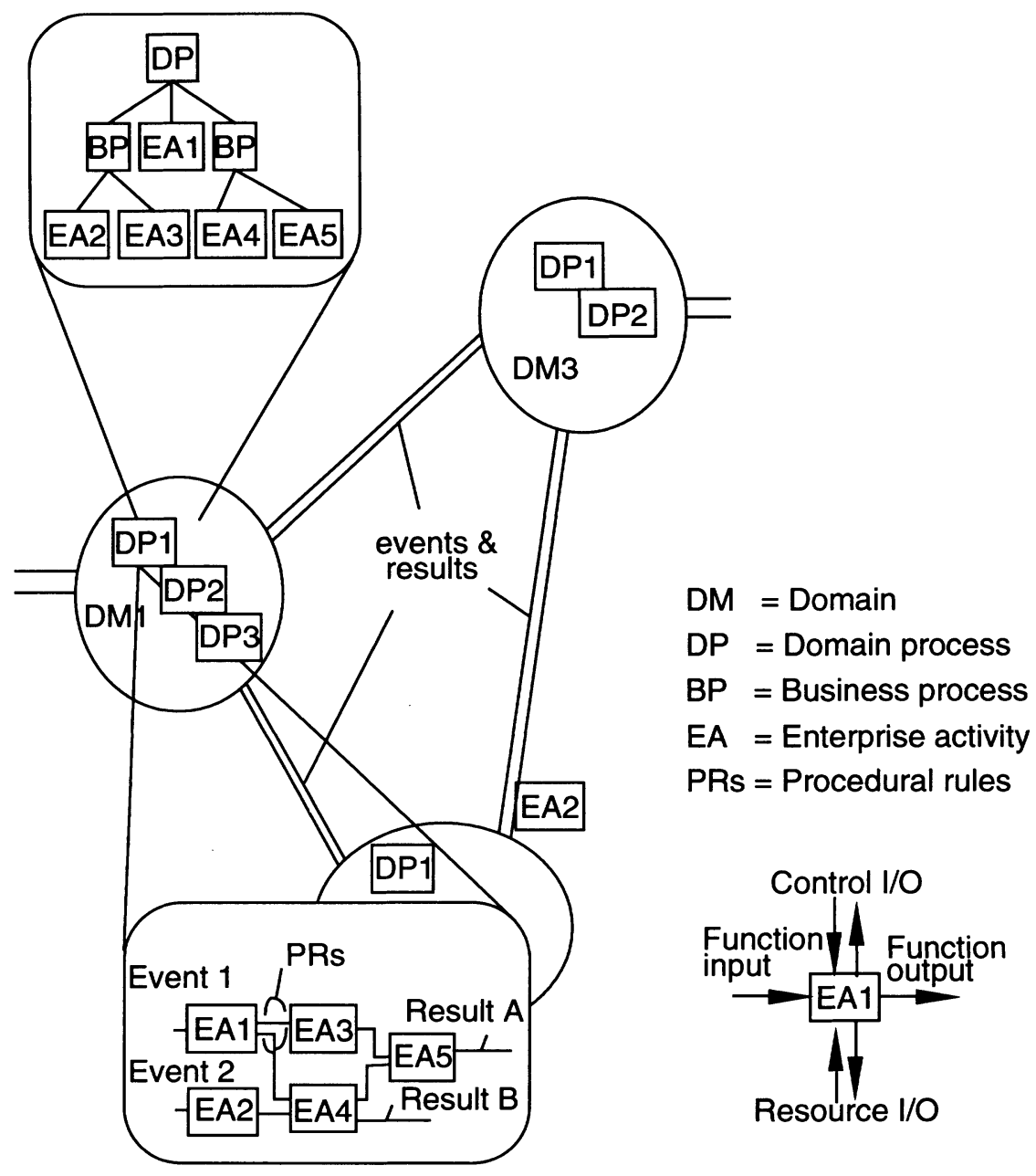

Figure 6.8 Internal structure of a domain process.

The CIMOSA modelling approach includes and extends previous functional modelling approaches [9] already in use in industry or being considered by standardization bodies. These recognize essentially only the concept of activity (also called function).

CIMOSA differentiates between 'as-is' and 'to-be' modelling. Modelling of an existing implementation will start with a bottom up description of the current operation. Abstraction from the 'as-is' description 
and modifying the operation would lead to a requirements and design of the 'to-be' model and analysis of its intended behavior. No specific methodology has been prescribed by CIMOSA thus leaving freedom for iterations as required between decomposition and aggregation as well as between modelling levels.

\subsection{CIMOSA SYSTEM LIFE-CYCLE AND ENVIRONMENTS}

To support its modelling concept, CIMOSA defines an enterprise system life-cycle and the corresponding environments in which the relevant lifecycle processes can be executed. These environments contain: (1) a dynamic definition (a model) of the life-cycle tasks in the form of a set of processes; (2) a set of resources providing the functionality required to carry out those tasks and (3) a set of common services providing system wide support for the execution of processes. This concept decouples the engineering of the enterprise's system(s) from the day-to-day enterprise operations.

In order to permit the processes for one task within the Product Lifecycle to be designed/updated without interfering with the execution of current operational tasks, CIMOSA provides two mutually independent, but interrelated, environments (see Table 6.1).

Table 6.1 CIMOSA life-cycle and environments

\begin{tabular}{lll}
\hline Life-cycle & Environment & Model executed \\
\hline system & enterprise engineering & $\begin{array}{l}\text { engineering } \\
\text { implementation model }\end{array}$ \\
product & enterprise operation & $\begin{array}{l}\text { particular } \\
\text { implementation model }\end{array}$ \\
\hline
\end{tabular}

1. The Enterprise Operational Environment in which products are developed, produced, marketed and sold, orders are issued and received, bills are paid and invoices submitted to customers in accordance with the processes defined for the enterprise's Product Life-cycles.

2. The Enterprise Engineering Environment in which particular enterprise domains are modelled (including the simulation/evaluation of alternative solutions), built, validated and released for operation in accordance with the processes defined for the enterprise's system life-cycle (except for the system operation phase). 
In both cases life-cycle processes are defined by a CIMOSA compliant model. In the case of the enterprise operations environment this model is user created so as to be unique to the particular enterprise. The model of the enterprise engineering environment is usually constructed by the IT vendor and then customized to the user's needs. Conformance to the CIMOSA Model Creation processes and the CIMOSA Model Consistency rules ensure that the end result is a CIMOSA compliant model (see also Table $6.2)$.

Table 6.2 Service entities of the Integrating Infrastructures service provided

\begin{tabular}{ll}
\hline Entity & Execution of enterprise model \\
\hline Business & According to business rules \\
Presentation & $\begin{array}{l}\text { Homogeneous access to humans, } \\
\text { machines, software applications }\end{array}$ \\
Information & $\begin{array}{l}\text { High level of consistent information } \\
\text { Common }\end{array}$ \\
System management & $\begin{array}{l}\text { System wide homogeneous data com- } \\
\text { munication }\end{array}$ \\
\hline
\end{tabular}

CIMOSA defines the basic phases of the System Life-cycle in terms which are independent of the modelling methodology employed. CIMOSA does not populate the System Life-cycle with a set of unique processes but leaves it open for tool vendors to define their own various proprietary methodologies in a CIMOSA compliant model. Thus alternative models (tool sets) will exist for the system life-cycle to reflect different modelling methodologies (e.g. waterfall, fast prototyping, concurrent engineering, etc.) for creating CIMOSA conformant enterprise models.

For example, the creation and execution of a CIMOSA compliant process involves the following basic tasks:

1. Identify the business domain

2. Select relevant building block types from a catalogue of CIMOSA building blocks 
3. Customize the selected types by adding enterprise specific parameters to make instances of the building block types

4. If required install and describe any additional resources

5. Release the enterprise model which contains the instances, into the operational system

6. Add, at execution time, technical parameters and logistic variables to a copy of the released instances (create occurrences).

CIMOSA proposes a formal engineering release of the enterprise model at the phase-over from the engineering to the operational environment. Changes to be made to the operational system have to be implemented, validated and documented in the engineering mode rather than in the operational mode. Only then can a sufficient engineering discipline be introduced into the enterprise operation and only then can model consistency be assured. This release process acts as a 'bridge' between the system and the Product Life-cycle.

It should be noted that emphasis in CIMOSA Enterprise Engineering is on model maintenance (update, modification, extension) rather than on model creation. The latter is seen as a one time affair, whereas the former is a continuous task for redefining the model contents. Only if the model continues to reflect the reality of the enterprise operation will the use of model based operation control and monitoring and decision support become a reality.

\subsection{CIMOSA INTEGRATING INFRASTRUCTURE}

CIMOSA copes with heterogeneity by providing the CIMOSA Integrating Infrastructure (IIS). This IIS provides a structured set of system wide services thereby avoiding redundancy of function in the enterprise's systems. By making use of these generic services the application systems in the CIMOSA Environments need no longer contain the specifics of the data processing environment. This provides increased portability and flexibility of the application systems and reduces the maintenance tasks considerably.

The IIS supports integration by providing homogeneous access to system components. The services of the CIMOSA Integrating Infrastructure address the problems of Application Integration. The IIS supports model execution and engineering through a set of five generic service entities (see Fig. 6.10). It also provides a unifying software platform to achieve integration of heterogeneous hardware and software components.

The CIMOSA Integrating Infrastructure is tuned to the execution of CIMOSA models. Triggering of domain processes invokes the Business 
Entity which will process the request and which will initiate the creation of a domain process occurrence and its subsequent processing by other services according to the nature of the event. The services of the Business Entity will schedule resources and execute functional operations through the Presentation Entity. It will also provide and remove information via the Information Entity. Communication across the entire system and the needs of system management are provided by the remaining two entities (Common and System Management Entity).

The AMICE project defines functionality of the IIS services but its implementation is under the discretion of the IT vendors. The IIS may be implemented according to any standard or proprietary solution which provides the expected functionality. Access to the different services is specified by interfaces or protocols. Therefore, depending on the degree of software integration, the IIS software may be a very small extension or a large software packet added to the vendor system software.

The IIS provides a structured set of common System Wide Services that will avoid redundancy of functions in any CIM system. By offering these services system wide in a uniform manner the basis for required integration is created. The role of the IIS is to provide the computer integrated part of CIM, meaning that the integration is achieved through Information Technology, i.e. computers.

The IIS provides for a set of specified services to control and integrate the definitions in the implementation model of a CIM architecture and the resources (humans, machines, applications, etc.) performing these functions. The services will:

1. Isolate the application programs from the data processing and business environment

2. Operate system-wide and not require the architectural modules using these services to have any knowledge of their location and/or distribution

3. Provide common protocols allowing the humans, machines and application programs to interact with the IIS in a uniform way.

The System Wide Services appear to the user of the service as a single service across all nodes of the system. The user doesn't have to know how and where the service is provided. Within the services of the IIS one can distinguish three sets of services.

Function related services include all those services that are related to enterprise functions. These services manage the control and execute the enterprise activities. They provide the Integration of Functions.

Information related services include services that support the information processing activities of the enterprise. They deal with locating, accessing, 


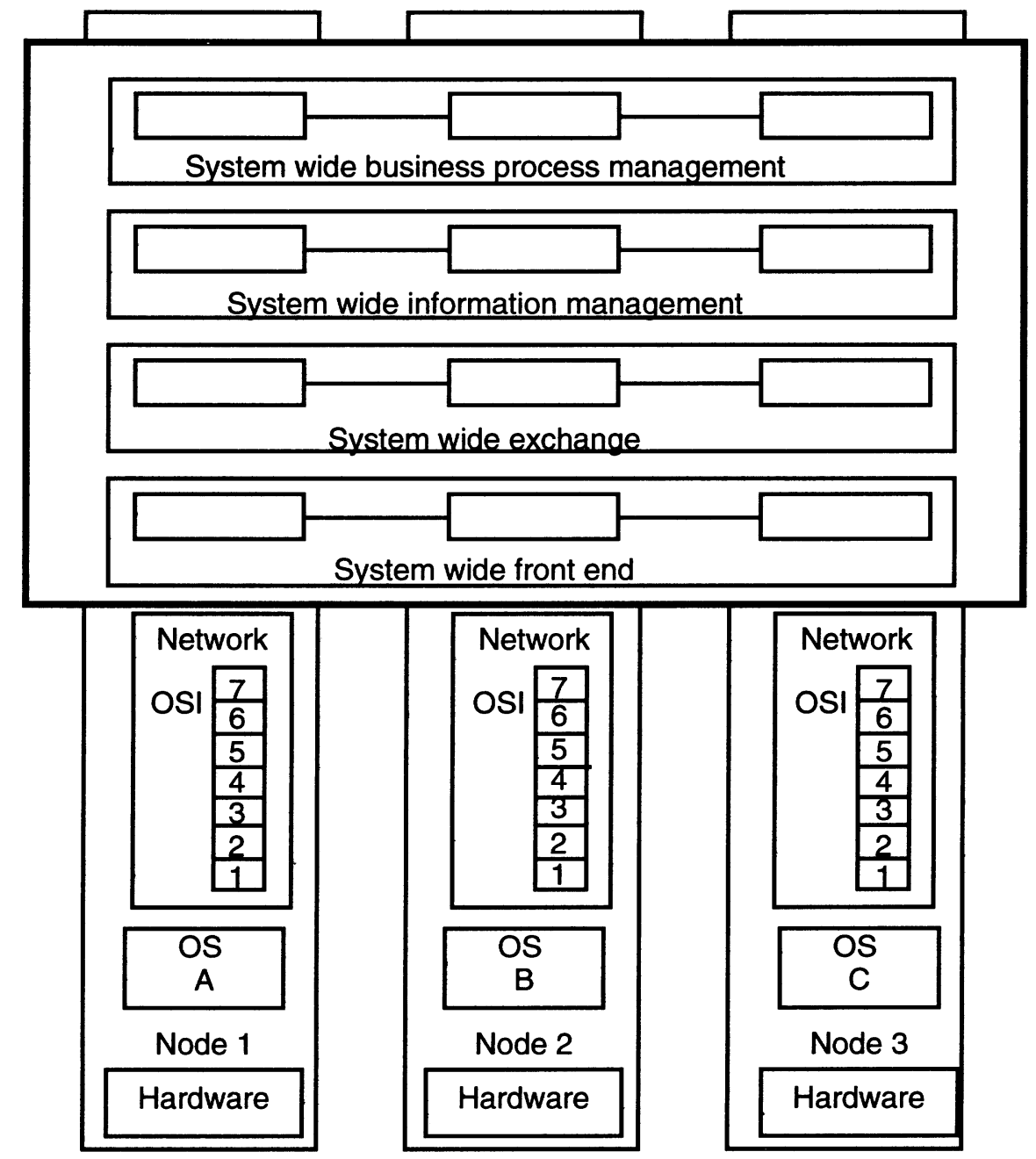

Figure 6.9 Overview of all the services within the IIS.

storing and maintaining the consistency of information. These services provide the Integration of Information.

Communication related services include the services required for the control of Intra- and Inter-System Communication. They provide the Integration of Communication.

Fig. 6.9 provides an overview of the IIS. The three sets of services that were first mentioned will be detailed hereafter. 
The Function Related Services provide a common functional control service that integrates all functions of the enterprise along business processes oriented towards the result of the enterprise. The system wide business process management services (see Fig. 6.9) uses both resource and organizational views of the architectural model and contains human entities, machine entities, and application entities which are interfaced to the architecture via System Wide Front End Services through front-end protocols (see Fig.6.10).

Three Management Services have been defined for the proper functioning of the enterprise:

1. The business process control services which manage and control the business processes of the enterprise on a system wide basis.

2. The Activity Control services which manage and control the execution of the activities in the enterprise on a system wide basis.

3. Resource Management services which use the resource information of the implemented model and occurrences of the business processes to schedule the resources and the business processes on a system wide basis.

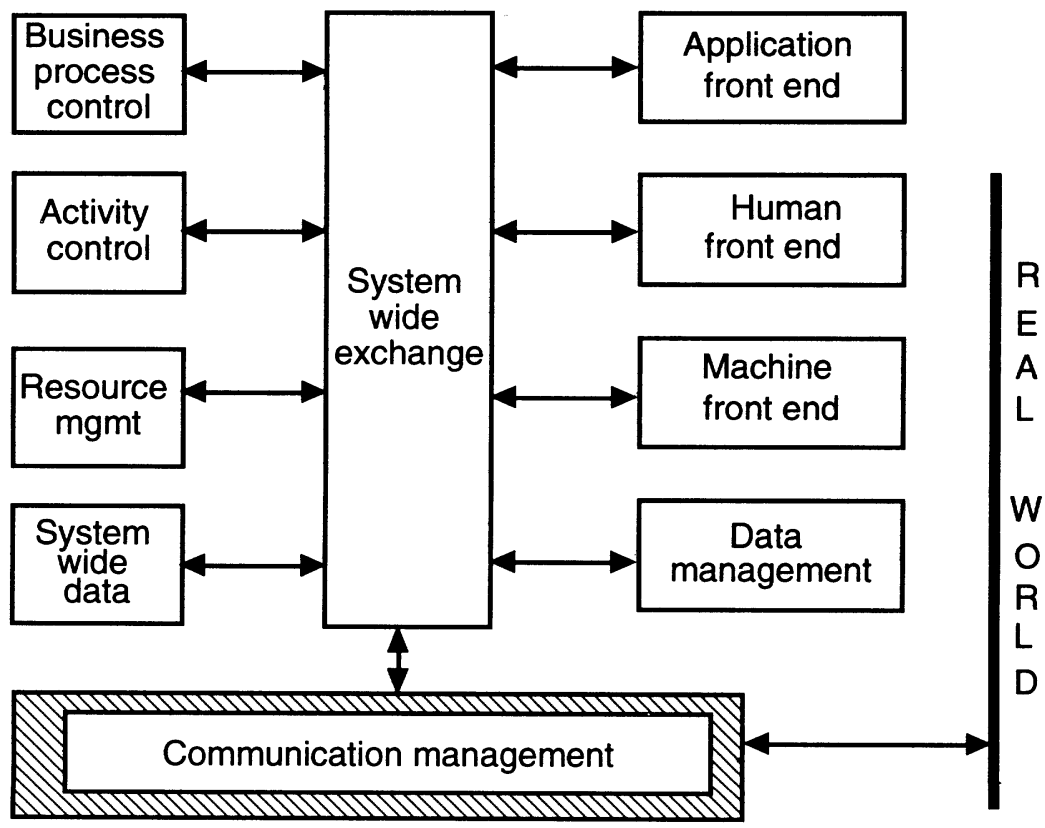

Figure 6.10 Services of the integrating infrastructure. 
The purpose of the Information Related Services is to manage the storage and retrieval of data on a system wide basis where the clients don't need to know how and where the data is stored. The services further provide system wide data consistency and information context.

The System Wide Data Service provides for the location of information, consistency of data, management of schema conversions and control of requests for data. It also provides for storage and retrieval of data through Data Management. The System Wide Data Services handle all requests for information from clients. The Data Management has only one client; the System Wide Data Services. Data Management interacts with the supporting functional entities (business entities, information entities, presentation entities and human and machine entities) for the storage and retrieval of data and caters for the further completion of the schema conversions.

The purpose of the Communication Related Services is to allow all implemented functional entities to communicate with each other on a system wide basis. These entities do not have to have any knowledge of where the other entity is located and how the locations communicate with each other. There is no difference between inter- and intra-system communication for the user of this service.

System Wide Exchange Services will provide an adequate service needed to implement access, agent and external protocols occurring among information related services and function related services, while factoring out as many as possible of the issues of synchronous communication.

Communication management, finally, has one client only: the System Wide Exchange Services. It interacts with the supporting implemented entities to send and receive data based on the concepts developed in the Basic Reference Model for Open System Interconnect (ISO 7498).

\subsection{APPLYING CIMOSA - A SUMMARY}

Fig. 6.11 illustrates the use of CIMOSA. The reference architecture provides the constructs (Generic Building Blocks) and standardized processes (Partial Models) which are used to create and maintain the particular enterprise model and to implement the IIS. The enterprise model describes the Product Life-cycle processes (domain processes) of the particular enterprise and their interrelations. These processes are composed of enterprise activities and functional operations. Resources are selected from vendor catalogues or from the installed enterprise base. 


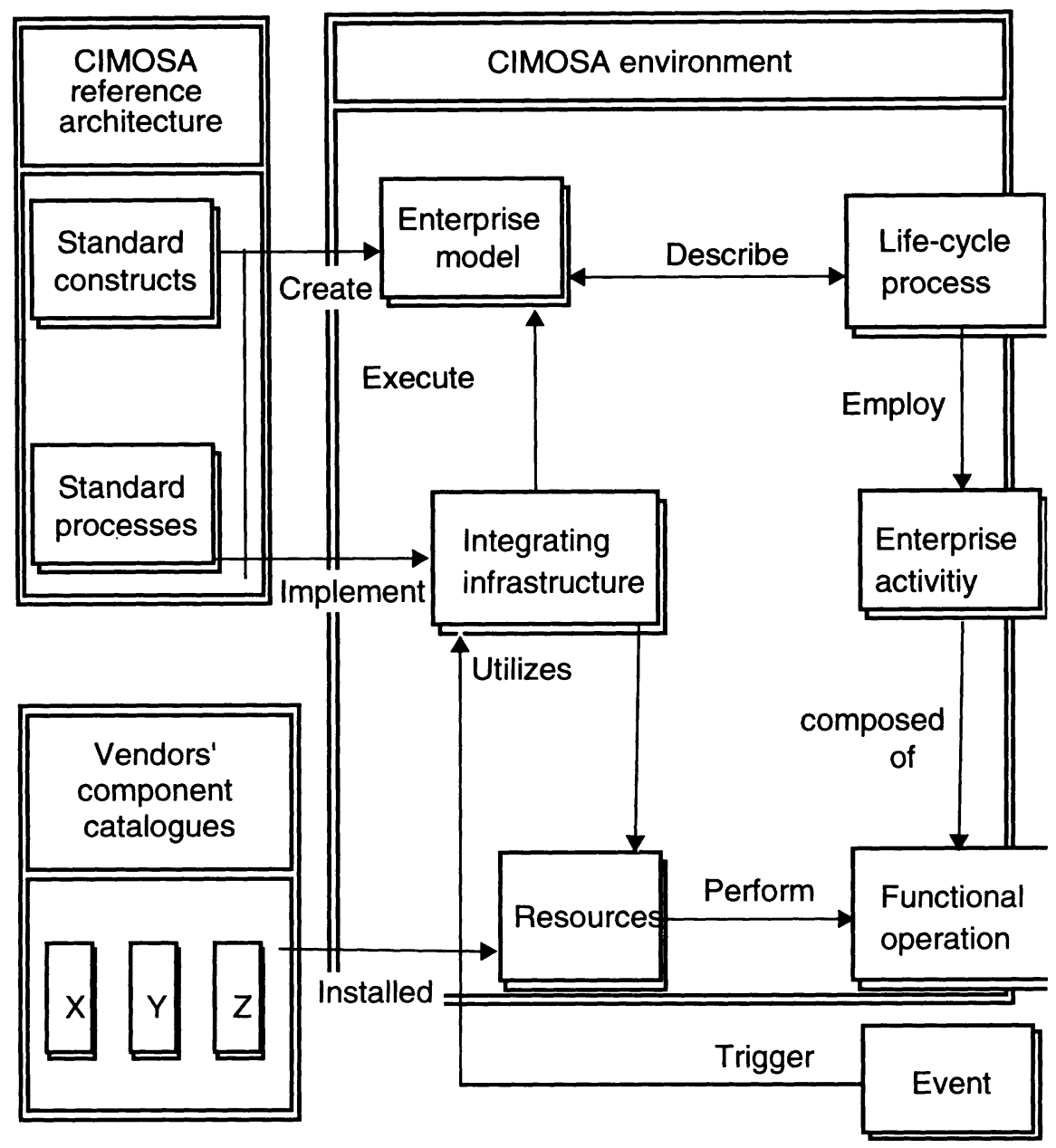

Figure 6.11 Creation and execution of CIMOSA models.

Model creation, implementation and maintenance are part of the engineering environment and follow the System Life-cycle concept. Model execution is concerned with the processes of type Product Life-cycle. These processes are performed through the utilization of relevant resources which are identified in the different processes as resource inputs of the enterprise activities. Model execution is initiated by events which represent real world happenings like receiving a customer order or identifying a due date. Any event will start execution of at least one domain process (see above). 
Model based operational control and monitoring requires a consistent and up-to-date model of the operation. Therefore, modelling has to be done by the people responsible for the operation and authorized to modify and adapt the particular part of the model according to negotiated and accepted needs and opportunities. Knowledge about interrelations and the impact of change have to be made known to the parties involved in the engineering phase and prior to release to operation. Therefore analysis and evaluation of change is an important part of model creation and maintenance and has to be employed by all the people involved in enterprise engineering. Assignments of responsibility and authority in the engineering environment are very important and have to be made with a clear understanding of the consequences involved.

\subsection{OVERALL SUMMARY OF ARCHITECTURE AND BASIC CONCEPTS OF CIMOSA}

Today the manufacturing industry is faced with a rapidly changing business environment. Varying market demands require frequent operational and organizational changes in the individual enterprise. CIMOSA therefore enables the user to describe his enterprise from two distinct standpoints:

1. The Business Environment as seen by the business user

2. The Physical Environment (the manufacturing and Information Technology implemented in the enterprise).

The prime goal of CIM and enterprise integration is to use Information Technology to integrate the enterprise operations. CIMOSA achieves this by linking the enterprise activities and functional operations into a dynamic state network, thus integrating the activities and the resources performing them. Fig. 6.12 summarizes the contents and use of CIMOSA in its two environments and the relations of its three major concepts with one another and the real enterprise world.

1. Modelling Framework

2. System Life-cycle

3. Integrating Infrastructure

CIMOSA provides a reference architecture in its modelling framework for the specific description of a particular enterprise. The architectural constructs of CIMOSA guide the CIM business user in the creation and maintenance process to obtain and maintain a consistent system description. These constructs will also guide CIM suppliers to develop, produce and market 
products which will fit the defined user requirements and comply with the implemented system.

The architectural constructs provided for the description can be viewed as the semantics for a manufacturing enterprise description language. This approach constitutes a completely new method for manufacturing system development. The method allows the end user to define, to prototype, to design, to implement and to execute the business according to his needs. The architecture also defines the necessary support environment for development and execution.

CIMOSA facilitates a System Life-cycle which guides the user through model engineering and model execution. Starting with the collection of business requirements in a Requirements Definition Model, the System Life-cycle carries the user through their translation into a System Design Model and finally, to the description of the implemented system (System Implementation Model). These phases are followed by a model release for operations and model execution for operational control and monitoring. To derive the system description all specific constraints of the particular enterprise are taken into account in the Design Specification Model. Model maintenance is a very important part of the System Life-cycle as well.

Model engineering and model execution are supported by the CIMOSA Integrating Infrastructure. The Integrating Infrastructure provides a set of generic services which process the released enterprise model and provide access to information, connect to resources and handle exceptions through reference to identified responsibilities and authorities. This infrastructure also hides the heterogeneity of the underlying Manufacturing and Information Technology.

The ESPRIT Consortium AMICE has developed CIMOSA and has validated its concepts in various case studies in its member companies. Validation work has also been done in other ESPRIT projects which will also lead to development of partial models in specific industry areas and particular modelling aspects. The project itself will further verify CIMOSA through industrial pilot implementation. Work on prepilots in enterprise modelling and model based operation control and monitoring has been started. Results from this work will become available in 1993. 


\subsection{REFERENCES}

[1] Anonymous, AMICE: CIMOSA, ESPRIT Project 5288, Milestone M-2, AD2.0 Volume 2, Architecture Description, Document RO443/1, Consortium AMICE, Brussels, Belgium, (August 24, 1992).

[2] H.T. Goranson. 1992. 'Dimensions of Enterpries Integration,' in [3] pp101-113.

[3] C. Petrie Jnr (Ed), 'Enterprise Integration Modeling,' Proc. First International Conference on Enterprise Integration Modeling, MIT Press, Cambridge, MA, USA (1992).

[4] CEN/CENELEC (The Joint European Standards Organization), European Prestandard Env 40003, 'Computer Integrated Manufacturing, System Architecture, Framework for Enterprise Modelling,' Berlin, Germany, (April 18, 1990).

[5] ESPRIT, Project 688, AMICE Open System Architecture for CIM, Springer Verlag, Berlin (1988).

[6] Publications by AMICE Project members

(a) Jorysz, H. R. and Vernadat, F. B., 'CIMOSA Part 1: Total Enterprise Modelling and Function View,' Int. J. Computer Integrated Manufacturing, Vol. 3, Nos. 3 and 4, pp. 144-156 (1990).

(b) Jorysz, H. R. and Vernadat, F. B., 'CIMOSA Part 2: Information View, 'Int. J. Computer Integrated Manufacturing, Vol. 3 Nos. 3 and 4, pp. 157-167 (1990).

(c) Klittich, M., 'CIMOSA Part 3: CIMOSA Integrating Infrastructure The Operational Basis for Integrated Manufacturing Systems,' Int. J. Computer Integrated Manufacturing, 3 (3-4) pp. 168-180 (1990).

[7] ESPRIT Consortium AMICE Open System Architecture, CIMOSA, AD 1.0, Architecture Description, ESPRIT Consortium AMICE, Brussels, Belgium (1991).

[8] A Set of General Works in Enterprise Integration Described in Numerous Publications.

(a) U.S. Air Force (no author) 'Integrated Computer-Aided Manufacturing (ICAM),' Architecture Part II, Vol. V - Information Modeling Manual (IDEF) AFWAL-TR-81-4023, Wright Patterson Air Force Base, OH, (June 1981). Plus other volumes in this same series.

(b) Throop, J. W. and Read, P. R., A Discrete Parts Manufacturing Model, Report Number R-83-SC-01, Computer Aided Manufacturing - International, Inc., Arlington, Texas (August 1983). 
(c) Simpson, J. A., Hocken, R. J., and Albus, J. S., 'The Automated Manufacturing Research Facility of the National Bureau of Standards,' Journal of Manufacturing Systems, Vol. 1, No. 1, pp. 17-32 (1982).

(d) ISO (International Organization for Standardization), ISO TC184/ SC5/WG1, The Ottawa Report on Reference Models for Manufacturing Standards, Report N51 (September 14, 1986).

(e) ESPRIT Pilot Project 34: Design Rules for Computer Integrated Manufacturing Systems.

(f) ANSA: Advanced Networked System Architecture.

[9] General works in enterprise modelling (numerous publications):

(a) Softech Inc., ICAM Architecture of Manufacturing, Integrated Computer-Aided Manufacturing (ICAM), Task I - Final Report, AFMLTR-78-148, Waltham, MA (November, 1978).

(b) Ross, D. T., 'Applications and Extensions of SADT,' IEEE Computer, Vol. 18, No. 4, pp25-34 (April 1985)

(c) Doumeingts, Guy, Méthode GRAI: Méthode de Conception des Systemes in Productique. These d'Etat Automatique, Universite de Bordeaux 1 (November 1984).

(d) Bravoco, R. R., and Yadav, S. B., 'Requirements Definition Architecture - An Overview',Computers in Industry, Vol. 6, No. 4, pp. 237251 (August 1985).

(e) Bravoco, R. R. and Yadav, S. B., 'A Methodology to Model the Functional Structure of an Organization, ' Computers in Industry, Vol. 6, No. 5, pp. 345-361 (October 1985).

(f) Ward, P. T., and Mellon, S. J., Structural Technologies for Real-Time Systems, Vol 1-5, Yourdon Press/Prentice-Hall, New York, NY (1985).

\subsection{FURTHER READING}

AMICE Consortium, Open System Architecture for CIM Research Reports of ESPRIT Project 688, Volume 1, Springer Verlag (1989).

Beekman, Dirk, 'CIMOSA: Computer Integrated Manufacturing - Open System Architecture, Int. J. Computer Integrated Manufacturing, Vol. 2, No. 2, pp. 94-105 (March-April 1989).

Canter, J. D., Vectoring Towards 2001, A Strategic Blueprint for the Competitive Enterprise,

CEN/CENELEC/WG ARC N150, Evaluation Report of Generic Constructs for Function Views and Other Views in ENV 40003, Brussels, Belgium (January 1992). 
Charue F., Apprentissages Organisationnells et Mutation Industrielle. Thèse, Universite de Paris (May 1991).

Chen, D. and Doumeingts, G., A Comparative Study Between CIMOSA and GIM, Laboratorie GRAI, University of Bordeaux, Bordeaux, France (May 1991).

Gallois,P.M., Le Manegement Industriel, Abance, Paris (1991).

Anonymous, Made in America, MIT Press, Cambridge, MA, (1989).

Roboam, M., La Méthode GRAI, Aerospatiale Paris, (1991).

Simon, H. A., Administration et Processus de Decision; Gestion Economica Paris, Paris, France (1983).

Tourratier, J. M., Les Résultats de l'Intégration Phase par Phase, ADEPA, Pari(1991).

Ullman, J. D., Principles of Database Systems, Second Edition, Computer Science Press, Rockville, MD (1982). 


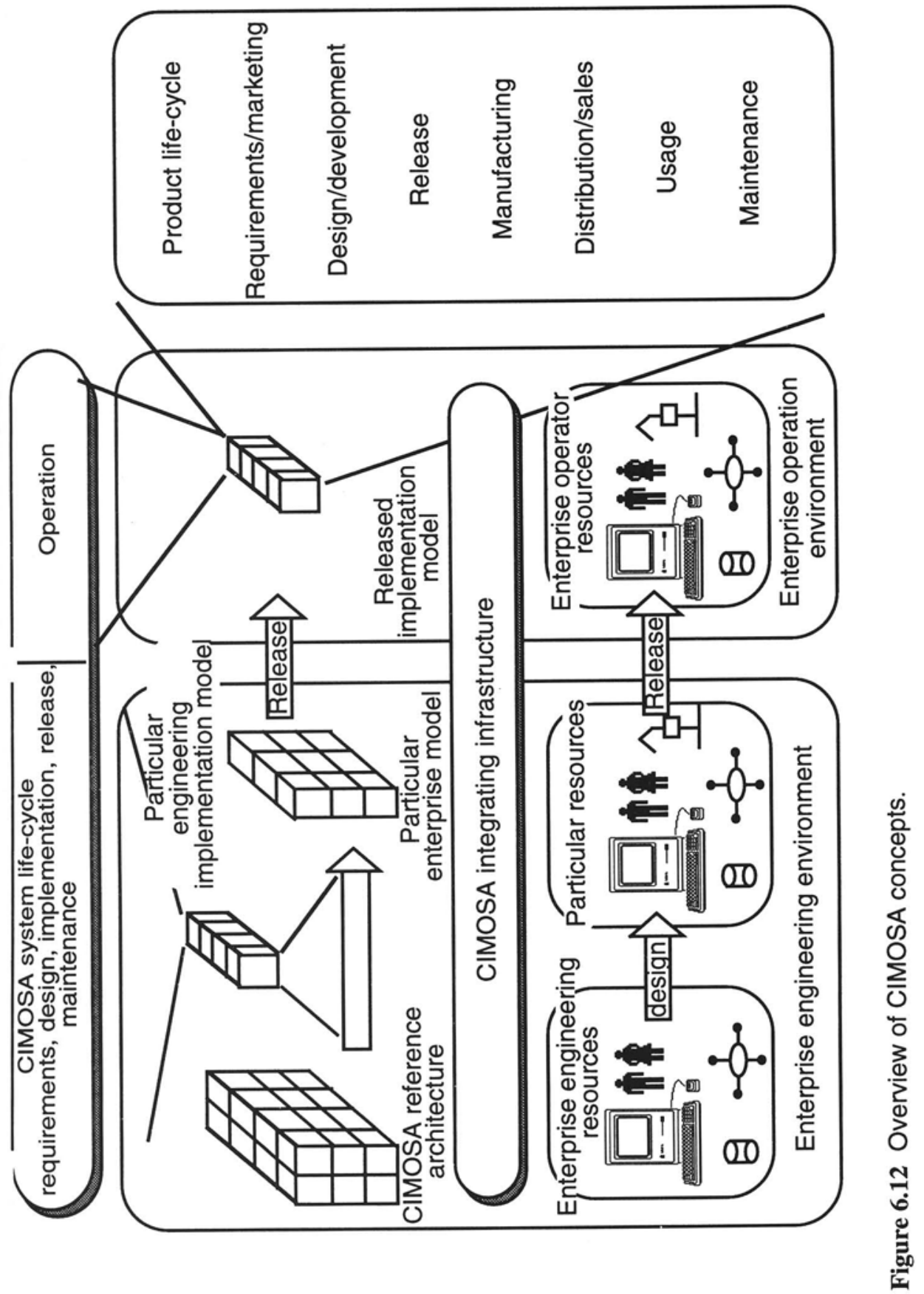

